\title{
Designing a Free Style, Indirect, and Interactive Storytelling Application for People with Aphasia
}

\author{
Elke Daemen ${ }^{1}$, Pavan Dadlani ${ }^{1}, \mathrm{Jia} \mathrm{Du}^{1}$, Ying Li ${ }^{1}$, Pinar Erik-Paker ${ }^{1}$, \\ Jean-Bernard Martens ${ }^{2}$, and Boris de Ruyter ${ }^{3}$ \\ ${ }^{1}$ User System Interaction \\ ${ }^{2}$ Industrial Design \\ Technische Universiteit Eindhoven, P.O. Box 513, \\ 5600 MB Eindhoven, The Netherlands \\ ${ }^{3}$ Philips Research, Eindhoven, The Netherlands \\ \{E.M.L.Daemen, P.M.Dadlani, J.Du, Y.Li, P.ErikPaker\}@tm.tue.nl, \\ J.B.O.S.Martens@tue.nl, Boris.De.Ruyter@Philips.com
}

\begin{abstract}
In this paper, we describe the iterative design and evaluation of a storytelling application for individuals with expressive aphasia. Our user studies show that besides basic requirements for medical care and training, there is an unmet need of aphasics to share their daily experiences and activities, anecdotes and feelings with their significant others. Thus, the goal of the proposed design is to enhance aphasics' quality of life by providing a platform for them to create and share their stories. More specifically, the goal is to enable them to play a more active role in social exchanges by providing them with a multimodal interface for storytelling that has the following functionalities: taking photos, making drawings and annotations, and recording sounds. In the end of this paper, we also summarize important design guidelines that surfaced during the course of this project and that are potentially relevant for other designers and researchers working with aphasics.
\end{abstract}

Keywords: Personal medical devices, aphasia, storytelling, multi-modal interfaces, user-centered design, iterative design, assistive technology, alternative and augmented communication, handheld devices.

\section{Introduction}

Aphasia is a communication disorder that is caused by brain injury or disease. Mostly it is caused as a result of a stroke that injures the language component of the brain [9]. Anyone can acquire aphasia, but most people who get affected are in their middle to late years. It is estimated that approximately 80,000 individuals acquire aphasia each year and about one million people in the United States currently have aphasia [10].

Depending on the area of the brain that is damaged, someone with aphasia can have impairments in speaking writing, reading or comprehending language. Expressive aphasia, also called Broca's aphasia, affects the production of language. Language is reduced to disjointed words, and sentence construction is poor. In extreme cases, aphasics may only produce a single word [10]. Receptive aphasia, also 
called Wernicke's aphasia, affects the receiving of language. For the aphasic, it seems that everyone is speaking in a foreign language she or he cannot understand and everything they read is gibberish [10]. In some cases, both the receptive and expressive language centers are impaired, which is called Global Aphasia. In this case, the individual has difficulty in producing and comprehending language.

Although the literature clearly defines different types of aphasia, in practicality aphasics are never completely of one type. Every aphasic has a unique speech language problem, so signs of impairment show up in a variety of ways in producing or understanding speech, reading or writing. In addition, aphasics vary in their physical and other cognitive abilities. For instance, many aphasics also have some degree of paralysis on their right-hand side.

The research documented in this paper investigates the needs of individuals with aphasia in different aspects of their daily life. This research motivated the design of a tool that supports aphasics in the creation and sharing of daily life stories. The research was conducted through an iterative process of designing and evaluating with both health-care specialists and aphasics.

\section{Related Work}

\subsection{Technology and Tools for People with Aphasia}

Gesturing, mimicking, pointing, drawing, and writing, are often used during aphasia therapy, while low technology aids are quite popular amongst aphasics. For instance communication books with written words, photos or icons [11] are commonly used because most aphasics prefer image-based information. Books are also cheap and can easily be carried around. The main disadvantages are their fixed vocabulary and the time taken to find what is needed.

Augmentative and Alternative Communication (AAC) devices are the major form of technology to support aphasics, and a lot of research and design has been done in this area. GPRS (General Packet Radio Service) systems, cameras and PDAs are all existing technologies that can be used for this purpose. Developments up to now have focused predominantly on devices to assist in direct communicative exchanges [8].

One of the most popular AAC devices is TouchSpeak [5]. It is a portable communication aid consisting of a PDA with specifically developed software. It supports multi-modality (i.e., images, sounds and text) by providing interfaces to a digital keyboard, a camera, a drawing pad, etc. To fulfill the requirements of different aphasics, speech therapists work together with an aphasic person to build a personal vocabulary and to select function modules. The flexible nature of the device is an especially strong feature, since vocabulary and functions can be adapted to the aphasics' needs. However, the software is installed on a PDA with limited screen size, which leads to a requirement for precise and accurate interactions. This obviously implies a limitation for the many aphasics who are physically impaired. In addition, the interface requires aphasics to browse into categories and layers, which is quite challenging for language-impaired people [1]. There are other devices that provide sentences to support simple conversations, such as ScriptTalker [5]. This device provides standard phrases of every-day situations, and it aims at aiding people with 
total loss of speech. It takes a lot of time and heavy cognitive efforts to construct new sentences, however.

Another popular tool is Lingraphica [5]. It is desktop software which aphasics can use to browse items within a virtually represented environment. The tool offers visual cues during the searching process. For example, if users want to drink milk, they will be presented with a home environment, they must open the door of the kitchen and a fridge will be there as a cue to help users find the milk. Once again, the need to browse into categories can pose a big challenge for the targeted users.

\subsection{Working with Aphasics}

Designing for people with aphasia is obviously non-trivial, since communicating with them is very difficult. Therefore, it is essential to involve expertise from several areas other than HCI. Several prior projects and research suggest that including psychologists, audiologists, speech therapists, social workers, or family members can help the design team to better identify the real needs of aphasics [2,6]. This is because these health-care workers and family members interact with aphasics on a daily basis, and are therefore able to better understand their abilities and problems within daily activities.

Participatory design methods have been applied in many aphasia research and design projects. Methods involving speech therapists as proxies in brainstorming sessions and interviews, and getting feedback of design concepts have been adopted, such as within the design of the ESI Planner [2], a daily planner for people with aphasia.

\section{User Study and Requirements Analysis}

\subsection{Contextual Interviews and Field Observations}

Given that it is a challenge to communicate with individuals with aphasia, we used alternative ways to communicate with them through their primary caregivers, speech pathologists, clinical linguists, and social workers who assist aphasics and their families. During face-to-face interviews with aphasics, they were able to interpret and validate questions, and express attitude and emotional responses towards our design concepts. The goal was to identify which aspects of their life are not well-supported through current tools and to understand their life needs in more depth than can be deduced from literature.

During the design process, we established a good working relation with a local Aphasia Union and we were invited to join meetings that took place every two weeks where more than 20 aphasics and their partners met and spend time together sharing stories, playing games, etc. We observed how aphasics communicate with their partners and with each other in a casual and relaxed context. We noticed that they use a lot of gestures to support what they want to express. Each of them comes to these meetings with a photo album that contains new photos of themselves, their family or friends. They use their photo albums to initiate a talk with caregivers and other aphasics. Moreover, they use tags with emotions and add them to different photos to help express their feelings. We found this very interesting and inspiring, since it reveals the important role of using pictures and emotion symbols in the daily 
communication of aphasics. Figure 1 shows an example of drawings used by an expressive aphasic trying to tell us what his earlier profession was. The drawing shows two swimming pools since he was a director of a swimming pool and the numbers represent the people who used to work with him. It is interesting to notice that some aphasics use letters to add to their drawings but mostly only the first letter of a word. For instance, they drew a box of milk and wrote the letter 'M' beside it.

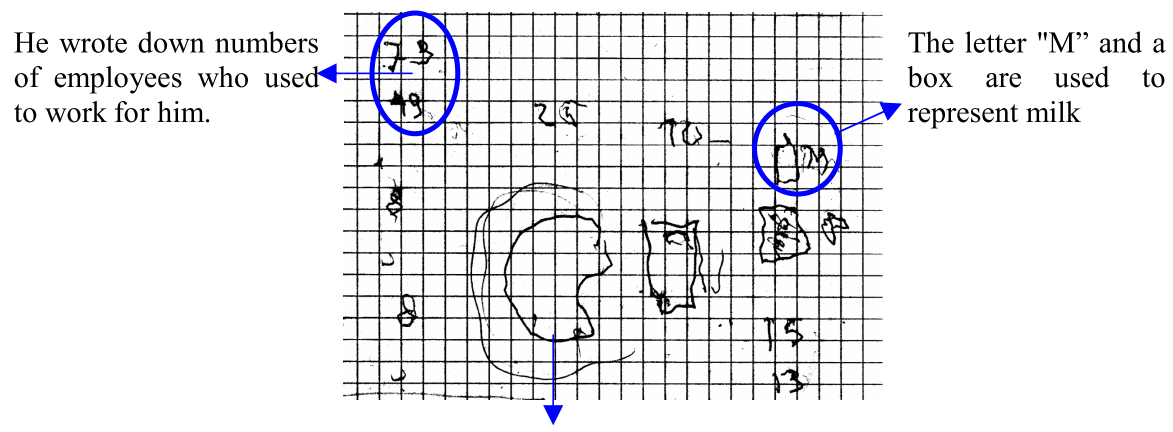

He drew a swimming pool to explain his previous profession

Fig. 1. An example of drawings made by an expressive aphasic trying to communicate what his earlier profession was

\subsection{Findings}

The study helped derive and prove the following needs of individuals with aphasia:

Like others, aphasic individuals want to be listened to and understood. Aphasics become nervous and get frustrated if others cannot understand what they are trying to express. This forces them to take a passive role in social interaction, which in turn increases the likelihood of developing stress and depression.

The proposed technology should not appear to be for disabled people. Although supporting devices are widely used by aphasic individuals, such as pictogram books, most aphasics would rather leave the device at home when they go to a social event. Ironically, many of them want to be social but choose not to communicate since their aiding device will emphasize their disability.

Like others, aphasics want to express their ideas, comments, anecdotes, and feelings about what occurred to them in their daily life. Although they have lost the ability to speak, there is a great need for them to communicate more about their emotions and daily activities with their significant others than only receiving daily care and help from the latter.

Aphasics require easy access and simplicity in their communication aids. Many aphasics fail to use some of the currently available technologies because these technologies do not adequately consider their capabilities. For example, the hierarchy structure used by TouchSpeak is confusing to many aphasic individuals because they find hierarchies difficult to understand. 
Aphasics need the ability to communicate at a distance instantaneously. Many aphasics stay at home alone. Their primary caregivers worry about this and usually try to arrange someone (neighbors, family, or friends) to be with them. Many aphasics get afraid of being alone since they may not be able to handle unexpected or emergency situations.

In addition to physical constraints, attention should be paid to psychological problems. It is of much interest to aphasic individuals to find ways to help relieve their stress and to become more connected to the world around them.

\subsection{New Insights}

Based on the above findings, there are two opportunities for supporting individuals with aphasia. The first one is a specific need to support synchronous distance-based communication to help aphasics deal with emergency and the second one is to enhance their quality of life by changing their passive position in their current daily communication. We decided to take the latter insight as the starting point for our design.

Current supporting tools, such as pictogram books or TouchSpeak, are mostly used for direct communication. For example, aphasics normally would request help and daycare like medication or getting food from their significant others. Aphasics would also use these tools to support their outdoor daily activities (e.g. shopping, meeting people, or making appointments). However, many of these devices make their disability apparent in a social situation. Hence, in direct communication aphasics tend to behave passively. They start to communicate only when they have to (e.g. getting food, drink, or medications).

However, when aphasics communicate in an indirect way, such as through writing e-mail, they have a more positive attitude. Like most people, they have time to prepare what messages they want to deliver asynchronously to others. Consequently, we want to focus on supporting indirect communication by means of a storytelling application which allows creating and sharing stories of daily life activities in a free personal style for people with expressive aphasia. The reason why we choose storytelling as a solution is because a large amount of people's conversation consists of sharing stories [3] related to anecdotes and events, and their attitudes towards them [4]. In addition, aphasics can make stories about their daily life, their hobbies, job, family, friends, etc., and they can share them with others.

Such a storytelling application has the potential of (1) changing the passive position in communication of individuals with aphasia, for example by initiating a conversation with family at the dinner table, (2) being able to share their feelings and attitude towards a particular topic or activity they have come across in their daily life, (3) capturing things they want to share with others, making the communication self-entertaining for them, and (4) relieving the stress and anxiety that aphasia brought to their lives.

\section{Iterative Design}

\subsection{Design Methods}

The user study helped us to clearly understand the diversity between aphasic individuals. Designing for all aphasic people is not possible, so a persona was created 
based on our user study. This helped us focus on a specific instance of expressive aphasia and guided our initial design. We conducted brainstorming sessions, which led us to four concepts of creating and sharing stories. These concepts emphasized different features relevant in the creation and sharing of stories. In order to present these concepts to experts and potential users, low fidelity prototypes were made and put into a storyboard with real use context.

\subsection{Initial Conceptual Interface Design of the Storytelling Application}

To achieve our goal to help aphasics tell a daily story and be able to share it, four concepts were developed for initial user evaluation. In each of the concepts, users are able to retrieve, create and view stories made by them. Each concept is identified by different features of creating and retrieving stories, icons, color schemes and graphical layouts (Figures 2 and 3).
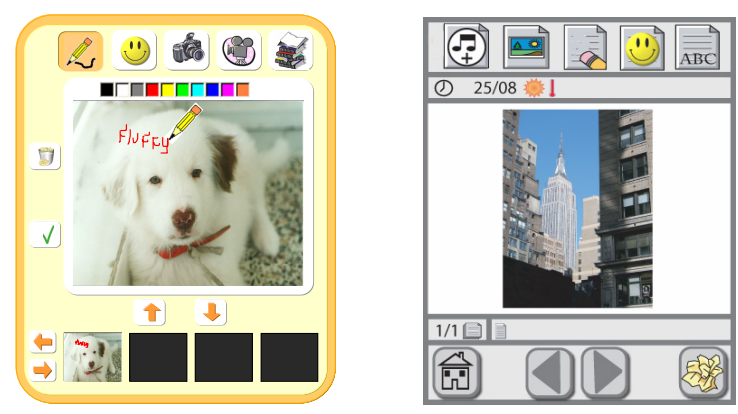

Fig. 2. Concept 1 (left) with a storyline and Concept 2 (right) where sharing stories is done manually with arrows
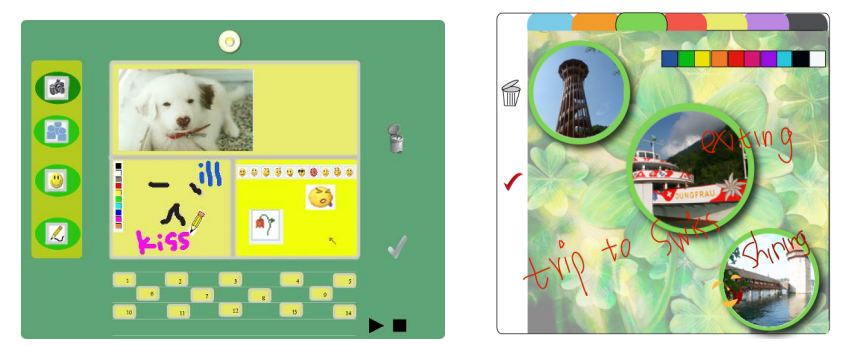

Fig. 3. Concept 3 (left) shows creating a clip with three components made of pictures, annotations and emotions, and Concept 4 (right) shows making a story as a one-page photo collage with annotations

\subsection{First Iteration: Testing Concepts with Speech Pathologists}

The first evaluation was carried out with four speech pathologists to validate the general concept of storytelling and get initial feedback of the conceptual interface designs described in 4.2. All experts agreed that the concept of storytelling is an 
unmet need for this population, and they envisioned the benefits of having such a tool for them.

The feedback provided us with valuable insights for further concept refining. First, in terms of physical capabilities of many aphasics, due to an impairment of the righthand side of their body, taking pictures with one hand is difficult for them. Often, their right-hand side vision is also affected. Thus, interface elements should be placed in the center or towards the left-hand side of the interface.

Second, in order to properly match available cognitive abilities, the interface should be as simple as possible and only support the basic features that are needed to create and share a story. The visual design should not be cluttered or too colorful, and interface elements should be obvious and easy to interpret. The navigation should be simple and the number of steps required to make a story should be kept to a strict minimum.

Third, about the features used in different concepts, sound recording was preferred over video recording. Besides, annotations would not be made to videos. Many aphasics have problems with the concept of time in terms of numbers and calendars. The people library was thought to be a practical feature for making stories, whereas the pictogram library could be too overwhelming.

Changes were made based on the feedback from speech specialists. Concept 4 (Figure 3) was dropped since it was considered too complicate, with too many colors, different photo orientations, and many collage templates. Features of the other three concepts were adjusted and integrated according to the feedback.

\subsection{Second Iteration: Testing Concepts with Aphasics}

Low fidelity prototypes that demonstrated all interaction steps for making and viewing a story were made in MS PowerPoint for the refined design concepts. These prototypes were evaluated with three aphasics and their partners from the Aphasia Union and the feedback is summarized below.

First, simplicity was considered to be important for both the graphical design and the interaction design. Amongst others, this implied that the number of steps needed for creating a story has to be kept to a strict minimum. Second, in terms of features, the storyline at the bottom of the screen of the first concept in Figure 2 seemed important when creating a story that contained several pictures. Users didn't find the keyboard necessary for making a story. They found adding sound to pictures a feature they might use, although it may be difficult for them to understand it properly. Taking pictures and adding annotations was an attractive feature to them, but being able to take pictures themselves could again prove to be a physical challenge.

\subsection{Third Iteration: Testing Interface Elements}

A list of different metaphors was created to represent each interface element. We asked four aphasics with their partners to choose the icons that were most clear to represent the intended function, such as drawing, emotions, taking pictures, etc. This helped finalize the concept prior to implementation. The participants liked those icons 
that were not abstract but more realistic. They preferred the $3 \mathrm{D}$ icons because they look more like real objects than the $2 \mathrm{D}$ icons.

\subsection{Fourth Iteration: Final Concept Prototyping}

A final concept was created using the feedback from speech specialists and aphasics. Users are provided with four input modalities for creating a story: taking pictures, drawing and annotating, adding emotions, and recording sound. Figure 4 shows the final interfaces of the application.

The first interface will show all the stories in chronological order with the latest being on top. An old story can be retrieved from this interface and a new story can be created by tapping on the blank one. The second interface is for editing stories. After taking a picture, users can add annotations, emotions, or sound to it. When their editing is finished they will save it (check button) as a clip of their story, which will appear in the storyline at the bottom. Users can navigate through the storyline manually and edit or delete (with the trash button) any clip of their story. The third interface is for playing stories, which can be by means of a slide show in the sequence of the storyline.
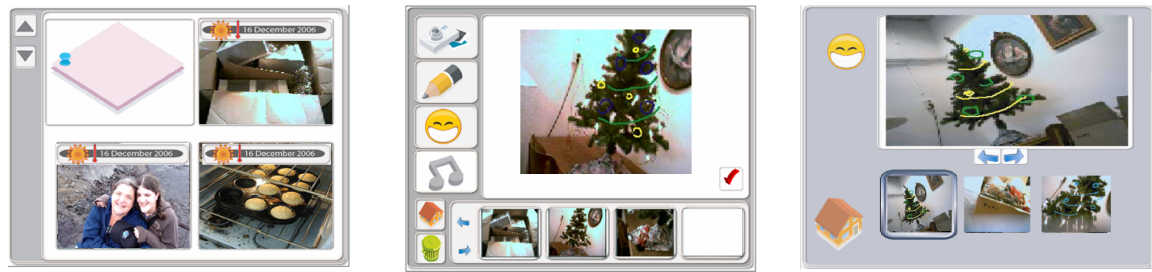

Fig. 4. The final concept has an initial screen to retrieve stories or to start creating a new story (left), while creating or editing storyline clips is done by means of an interface that offers four input modalities: pictures, annotation, emotions, and sounds (middle). Finally an interface is made for showing and browsing the story created by a user (right).

We presented users a slideshow of different ways they can tell a story to someone. The basic method is to show the story directly to others, but we took a step further and showed other ways, such as connecting it to the TV to show family members and friends, or sending a story via e-mail. When spouses are at work, they worry about leaving them at home without knowing how they are doing. Sending stories via e-mail could tackle this problem. These are opportunities of expanding the storytelling concept, and the feedback was positive.

\subsection{High Fidelity Prototype}

The storytelling application was developed in Macromedia Director and prototyped on the Motion Computing LS800 8.4" Tablet PC. An external webcam was fixed to support picture taking (Figure 5). 


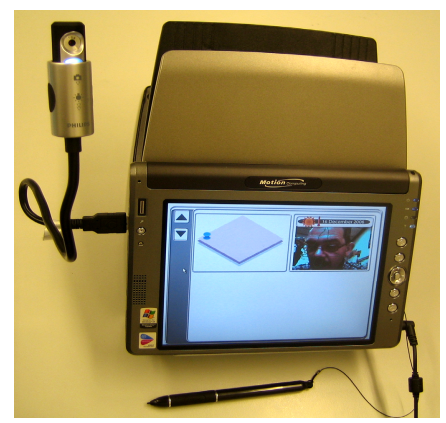

Fig. 5. The storytelling application in a Tablet PC with a webcam attached to it

\section{Evaluation of the Storytelling Concept}

Our next step was to conduct an evaluation of the storytelling prototype. The objective of this phase was to validate our concept of storytelling for aphasics with target users, and to decide whether or not the proposed tool could potentially influence their lifestyle. We wanted to meet our initial requirements such that individuals with aphasia can (1) construct, record, and share their life stories and anecdotes with family and friends, (2) prepare their story with not too much cognitive effort, (3) carry and operate the prototype within their physical capabilities, and (4) express their feelings and thoughts on subjects of interest.

\subsection{Participants}

A pilot experiment was done with an elderly person to validate the testing protocol and make changes to the procedure. Five aphasic individuals ( 4 males and 1 female) and their primary caregivers (their spouse in one case and speech therapists in the others) participated in this evaluation.

We selected participants with different levels of expressive aphasia. Two had a severe type of expressive aphasia. They could say 'yes' and 'no' to answer questions and could say their own names. Two other participants had medium level of aphasia. One of them could use several words and write some. He also could use drawings on a notebook to make clear what he meant. Another participant could use many words but changed some as he spoke. He could write a little and could read some words. Three of them were totally right side paralyzed and were in a wheelchair. The other two patients have problems using their right arm.

\subsection{Methodology}

Procedure. Observations, interviews, and questionnaires were adopted as main methods for testing [12]. The sessions took place at the participants' home or in a local rehabilitation center. Each session took approximately 60 minutes. Participants, together with their spouses or speech therapists were explained the storytelling prototype by means of a video prototype. It showed them a scenario of when and how the prototype can be used. The video demonstrated the complete process using all the 
features and playing back the story to a significant other. Participants were then given the opportunity to explore with the prototype and clarify any doubts.

We asked participants to create a short story about anything they would like to by taking pictures and using the storytelling features, and later sharing this story with their spouse or speech therapist. Since it is not possible to get their feedback directly, observations were made of facial expressions and physical signs of confusion or enjoyment, which could be used to identify their attitude and opinion about using the prototype. After the test, participants were asked to rate a set of 36 statements in terms of agreement in the form of five smileys ('very sad' to 'very happy', to represent 'strongly disagree' to 'strongly agree'). These predefined rating scales are already being used with aphasics for other purposes in rehabilitation centers. Since the goal is to make the communication between aphasics and significant others more interactive, the opinion of caregivers is essential and interviews were made with them at the end of the session.

Measures. Qualitative measures were used for evaluation. Our main purpose was to evaluate the acceptance of the storytelling concept and of the features in the prototype. In addition to this, physical use of the prototype, ease of use and graphical design were also tested.

\subsection{Results}

Storytelling. In general they liked the concept of storytelling. Participants indicated that our design is useful for them to tell stories to their significant others. They thought the system was easy to learn and use. They would use the system regularly to make and tell stories to their family or friends, and in fact they believed our system is better than their current ways of communication. They indicated that they would use the system more for recording daily activities. Although the storytelling application is to support indirect communication, they found it useful and would use it to communicate directly as well.

The partners and speech therapists also indicated that storytelling is a good concept and would help aphasics and their partners tell their daily activities. It would enhance their quality of life together because when the partner comes back from a long day of work, they can sit together and share stories of the day. The therapists also indicated that the designed interface is very simple and could be understood and used by a lot of people with expressive aphasia.

Features. The impression about the features was different for each aphasic user, but this is due to their different types of expressive aphasia. We asked participants to rank the four features in order of importance using cards with the icons and names of each. After this, we gave them cards of additional features such as a pictogram library, a people library, a movie maker, a keyboard, etc. They were asked to indicate which additional feature they wanted and how important they considered them to be in comparison to the current features. They all ranked the four current features as most important, but they wanted some additional ones and this differed based on the level of expressive aphasia they had. This does demonstrate that features should be customizable for individual needs. 
The first user indicated that she liked the emotions feature the most. This is because she currently finds it hard to express her feelings and the smileys helped her express how she felt. She indicated that the drawing feature is her second choice and drawing could also help her express her thoughts. When she was creating the story she made a picture of one of the observers and drew a house next to him. Later, when she was telling the story to her spouse, she indicated that the observer was visiting her house. The sound feature was the least appealing to her, since according to her it would not add much to the stories she would make. In addition to the current features, she wanted a pictogram library where she could select icons to add to her story.

The second user indicated that adding sounds to pictures was the most important feature for him. This is because he has a medium level of aphasia, and most of the times he needs to hear the first part of a word to instruct him to say it, so for him sound is very important for communication. In addition, the drawing tool was the second most important feature. He could make drawings of what he wanted to say when he wouldn't be able to find the words he needed. This participant liked the emotions feature the least because he already can express his feelings very well. He wanted the keyboard as an additional feature so he could make annotations in advance to help him tell the story.

The third participant, who had a light form of expressive aphasia, also liked the sound the most because it could help him retrieve words. Similarly to the second participant, he liked the drawing feature because it could also help him express his thoughts when he could not find the proper words. This participant did not want any additional features and found the current ones enough to create stories. The last two participants ranked taking pictures the highest and valued the importance of it when creating stories. One of them preferred sound over emotions and drawing because he could utter some words and sound could help him utter the right word, whereas the other participant preferred drawing over emotions and sound, because he could only utter 'yes' and 'no'. The latter preferred having a keyboard as an additional feature since his reading capability was not completely lost.

Prototype. Our main focus was to evaluate the storytelling concept rather than the physical product. However, based on the observations and feedback, it is essential that such a device be designed specifically for aphasics rather than using an off-the-shelf platform like our tablet. All patients indicated that the screen of the Tablet PC was big enough and although they indicated that the pen was easy to use, they all required a fair amount of training to use it properly. The storytelling device should consider the ergonomic factors of such individuals. The device was not easy to hold because all participants have an impaired right-arm and three of them use a wheelchair, so being able to hold the device with one hand and write with the other (left) was difficult for all the five participants.

\section{Implications}

Having gone through the user-centered design process for designing a storytelling application for people with aphasia, we feel we can also contribute some worthwhile insights that might have implications for related work. Several recommendations have 
been derived and divided into three broad categories: working, designing, and evaluating with individuals with aphasia.

\subsection{Working with Individuals with Aphasia}

Proxies are indeed important to be involved in the participatory design. We followed similar methods used by previous research to interact with aphasics by means of proxies [2]. Involving proxies to not only help communicate, but also to gather aphasics' needs and obtain their feedback about the designed product is valuable for designing for aphasics. In addition, social workers who understand many of the difficulties that aphasics and their families go through can provide key information about their real needs.

Use closed questions. Many aphasics can answer yes and no, so well designed closed questions are important for getting direct feedback.

\subsection{Designing for Individuals with Aphasia}

Start designing for a Persona. There is a wide variety of aphasics. Not all expressive aphasics have the same problems, and it is impossible to design for all of them. It is important to create a persona of a specific aphasic, make an initial design, and then iteratively adapt the design for a larger user group.

Simplicity is essential in interactive technologies for aphasics. The interface must be simple in all interactive dimensions. Designing abstract elements for aphasics should be avoided. The number of steps required to do a particular task should be strictly kept to a minimum. Metaphors used for icons should be as representative as possible of the intended meaning. The interface should not be too colorful or too cluttered, since this distract them and they can loose their focus of attention. Semantic categorizing should be avoided [1] and interface elements should be kept as visible and accessible as possible.

Structure and layout of the interface. Interface elements and navigation tools, should be big enough to be identified. Due to impairments on the right-hand side of their body, their right-hand side vision may be affected. For those who have this problem, anything placed on their right-hand side will not be identified as being present until it is pointed out to them. It is suggested to place interface elements either in the center or at the left-side of the interface.

Use of accessible and portable technology. The designed product should consider the ergonomic factors for those aphasics with physical disabilities on the right-hand side of their body. Aphasics should be able to use the product with one hand (typically with their left hand), hence, it should not be heavy or bulky.

When using touch screens, it is important to get the calibration and sensitivity right. Users may end up tapping on the wrong interface element leading to undesired results. From previous research, users preferred interacting with fingers and being able to concentrate on pointing only, rather than gripping (e.g. a stylus) and pointing [7]. In addition, tapping interaction has shown to be problematic for those with motoric impairments as it provides no support for targeting [8]. 


\subsection{Evaluating with Individuals with Aphasia}

Provide adequate time for evaluating with aphasics. The evaluation sessions took around an hour per participant and it was observed that half-way through, users would tend to loose confidence and become impatient. They may loose track of the goal of the session. Evaluation sessions should be broken up into shorter ones. In addition, experimenters should constantly confirm if participants are on track as they move on with the session, since aphasics may just say 'yes' to everything when they don't really mean it.

Provide detailed guided training prior to evaluating the product. Often, in evaluation methodologies, participants are given some time to explore by themselves the product after being instructed on how to use it and prior to the testing session. When evaluating with aphasics, however, a self-exploratory phase is not possible. Instead, the experimenter has to explore together with the participant, guiding them through step-by-step each interface element, and often having to repeat several times.

Add context to demonstrations. To help aphasic users understand how the product works, scenario based context should be added to low fidelity or video prototypes. On the other hand, high fidelity prototypes should look as real as possible, since aphasics may not understand the limitations of a prototype. For example, one of the users did not like the camera function very much since the picture quality processed by the software was not very good.

Use symbol-based rating scales. Rehabilitation centers use defined rating scales in terms of smileys which work very well with individuals with aphasia.

\section{Conclusions and Future Work}

We have presented a description of the process we followed for designing an interactive system that can support storytelling for people with aphasia. We initially made field observations and interviews to identify their needs. We further explored the concept of creating and sharing stories with significant others and iterated through low fidelity prototypes and evaluated the final concept in a working prototype. From our user study we learned that storytelling is an unmet need of individuals with aphasia that current technologies do not support and our final concept will help them (1) change their current passive position in a social environment, (2) express their ideas, thoughts, and feelings about a chosen subject of interest, and (3) support their communication with significant others.

We believe that an additional longitudinal field test is required where the prototype is kept with potential users for a period of time without any experimenters around, and have the device as part of their daily life. This can demonstrate its actual value in real life settings even after the novelty effect wears off. At the end of the trial time we will collect the same information and assess the feedback. In addition, we made an initial exploration of different ways for aphasics to show their story and we can conclude that there is an opportunity to have users share their stories in different modalities and support distance-based indirect communication. 
Acknowledgments. Rehabilitation Centers in Blixembosch, Rotterdam, and Ghent, the Emilius School, the Eindhoven Aphasia Union, and Marleen Tournel for all their support. We would like to extend our gratitude to all the aphasics and their spouses for their valuable time and feedback. Many thanks to Ankun Liu for all his help with our prototype.

\section{References}

1. Ahlsén, E., et al.: Virtual reality as a commnunication aid for persons with aphasia. In: Proceedings Disability, Virtual Reality \& Associated Technologies, Sweden, pp. 229-235 (1998)

2. Boyd-Graber, et al.: Participatory Design with Proxies: Developing a Desktop-PDA System to Support People with Aphasia. In: Proceedings CHI 2006, pp. 152-160. ACM Press, New York (2006)

3. Dunbar, R.: Grooming, gossip, and the evolution of language, Mackays of Chatham, Chatham, Kent, UK (1996)

4. Emler, N.: Gossip, reputation and social adaptation. In: Goodman, R.F., Ben-Ze'ev, A. (eds.) Good Gossip, pp. 117-138. Kansas University Press, Lawrence, KS (1994)

5. Kitzing, P., et al.: Communication aids for people with aphasia. Proceedings Logopedics Phoniatrics Vocology 30, 41-46 (2005)

6. McGrenere, et al.: Insights from the Aphasia Project: Designing Technology For and With People who have Aphasia. In: Proceedings CUU 2003, pp. 112-118. ACM Press, New York (2003)

7. Moffatt, K.: Designing Technology For and With Special Populations: An Exploration of Participatory Design with People with Aphasia. University of British Columbia (2001)

8. Moffatt, K.: The Participatory Design of a Sound and Image Enhanced Daily Planner for People with Aphasia. In: Proceedings CHI 2004, pp. 408-414. ACM Press, New York (2004)

9. The American Heart Association: Aphasia (December 2006), Available at http://www.americanheart.org/presenter.jhtml?identifier $=4485$

10. The National Institute on Deafness and Other Communication Disorders: Aphasia (December 2006), Available at: http://www.nidcd.nih.gov/health/voice/aphasia.htm

11. Van de Sandt, et al.: High-tech AAC and aphasia: Widening horizons? Aphasiology, pp. 453-469 (2000)

12. Nielsen, J.: Usability Engineering. Academic Press, Boston, USA (1993) 